\title{
Expression of FAS-L Differs from Primary to Relapsed Low-grade Gliomas and Predicts Progression-free Survival
}

\author{
JAN-MICHAEL WERNER ${ }^{1}$, SASKIA KUHL ${ }^{1}$, PANTELIS STAVRINOU ${ }^{1}$, GABRIELE RÖHN ${ }^{1}$, \\ BORIS KRISCHEK $^{1}$, TOBIAS BLAU ${ }^{2}$, ROLAND GOLDBRUNNER ${ }^{1}$ and MARCO TIMMER ${ }^{1}$ \\ ${ }^{1}$ Department of General Neurosurgery, ${ }^{2}$ Institute for Neuropathology, \\ Center for Neurosurgery, University Hospital Cologne, Cologne, Germany
}

\begin{abstract}
Background/Aim: The tumor necrosis factor FAS is overexpressed in high-grade gliomas (HGG). Only little is known about FAS or FAS ligand (FAS-L) in low-grade gliomas ( $L G G)$. We explored FAS/FAS-L expression in $L G G$, focusing on differences in primary and relapsed $L G G$ and on its prognostic value. Patients and Methods: A total of 133 glioma samples ( $73 \mathrm{LGG}, 60 \mathrm{HGG}$ ) were collected. The $L G G$ samples included 15 matched pairs of primary and relapsed tumors. RT-PCR was performed to measure FAS/FAS-L expression, using subunit A, flavoprotein variant (SDHA) as housekeeper. Clinical data included progression free-(PFS) and overall survival (OS). Results: $L G G$ showed significantly lower FAS but higher FAS-L expression than $H G G$. The FAS-L expression was higher in primary compared to relapsed $L G G$ and had a positive prognostic value concerning PFS (median 45.20 vs. 31.37 months). Conclusion: FAS-L could act as a prognostic marker and potential target in primary $L G G$.
\end{abstract}

Gliomas represent the most common primary brain tumors. Recent data show a rise in the incidence of gliomas to 5.9 per 100,000 inhabitants with an estimated annual rise of $0.7 \%$ (1).

Low-grade gliomas (WHO grade II) tend to malignant progression. While progressing, the overall survival decreases from 5-10 years to approximately 12-15 months in secondary glioblastomas (GBM) (2). Molecular profiling of initial and recurrent tumors gains importance in diagnosis

Correspondence to: Dr. Marco Timmer, Department of General Neurosurgery, Center for Neurosurgery, University Hospital Cologne, Kerpener Strasse 62, 50937 Cologne, Germany. Tel: +49 022147882802, Fax: +49 02214786257, e-mail: marco.timmer@ukkoeln.de

Key Words: Glioma, FAS, FAS-L, low-grade glioma, LGG, CD95, CD95L. and novel treatment possibilities of gliomas (3). Isocitrate dehydrogenase $(I D H)$ mutation is a driving force in the malignant transformation, a diagnostic molecular marker for secondary GBM and found in $90 \%$ of low-grade gliomas (4, 5). Apart from $I D H I$-mutation status, the outcome factors of low-grade gliomas comprise the extent of resection, age, neurological symptoms, tumor size and location $(6,7)$.

The cell surface receptor FAS (Apo-1, CD95) and its ligand FAS-L (CD95L, CD178) are members of the tumor necrosis factor (TNF) family and are located on the human chromosomes 10 and 1, respectively (8). Ligation of FAS and FAS-L or targeting FAS with an antibody leads to apoptotic cell death, mediated by FAS-associated death domain (FADD) and activation of caspase 8 (FLICE) and caspase $3(9,10)$. FAS and FAS-L dependent cell death has been studied in human gliomas. Synthetic FAS ligands can drive glioma cells into apoptosis and induce cell death in glioma stem cells $(11,12)$. FAS expression in astrocytic gliomas correlates with higher malignancy (13). While FAS expression does not show prognostic value, an association of semiquantitatively analysed FAS-L expression and prognosis in higher grade glioma (HGG) patients was assumed (14, 15). Current research mainly focused on FAS and FAS-L in HGG and GBM. Only little data on FAS/FAS-L in low-grade gliomas is available.

The goal of this study was to explore the roles of FAS and FAS-L expression in low-grade gliomas. We were particularly interested in studying differences in primary and relapsed gliomas, evaluate the prognostic value of FAS and FAS-L and, additionally, increase the understanding of FAS and FAS-L in malignant progression.

\section{Patients and Methods}

Samples. The tumor samples were obtained directly from surgery, and immediately frozen in liquid nitrogen and stored at $-80^{\circ} \mathrm{C}$ until RNA extraction. The samples were collected between the years 1991 and 2014. Patient survival data were last updated on 01.01.2017. Non-tumorous tissue was obtained during the approach 
Table I. Glioma patient specifics: Summary of the patient specific data of 133 glioma samples included in the study, featuring data on histology, gender, age and tumor side.

\begin{tabular}{|c|c|c|c|c|c|}
\hline & $\begin{array}{c}\text { GII glioma } \\
\text { Samples (\%) }\end{array}$ & $\begin{array}{c}\text { GIII glioma } \\
\text { Samples (\%) }\end{array}$ & $\begin{array}{c}\text { Secondary GBM } \\
\text { Samples (\%) }\end{array}$ & $\begin{array}{c}\text { Primary GBM } \\
\text { Samples (\%) }\end{array}$ & $\begin{array}{c}\text { Total } \\
\text { Samples }(\%)\end{array}$ \\
\hline Number & 73 & 20 & 20 & 20 & 133 \\
\hline \multicolumn{6}{|l|}{ Histology } \\
\hline Astrocytoma & $34(47 \%)$ & $15(75 \%)$ & $0(0 \%)$ & $0(0 \%)$ & 49 \\
\hline Oligoastrocytoma & $28(38 \%)$ & $5(25 \%)$ & $0(0 \%)$ & $0(0 \%)$ & 33 \\
\hline Oligodendroglioma & $11(15 \%)$ & $0(0 \%)$ & $0(0 \%)$ & $0(0 \%)$ & 11 \\
\hline Glioblastoma & $0(0 \%)$ & $0(0 \%)$ & $20(100 \%)$ & $20(100 \%)$ & 40 \\
\hline \multicolumn{6}{|l|}{ Gender } \\
\hline Female & $34(47 \%)$ & $5(25 \%)$ & $7(35 \%)$ & $6(30 \%)$ & $52(39 \%)$ \\
\hline Male & $39(53 \%)$ & $15(75 \%)$ & $13(65 \%)$ & $14(70 \%)$ & $81(61 \%)$ \\
\hline \multicolumn{6}{|l|}{ Age } \\
\hline$<40$ years & $38(52 \%)$ & $7(35 \%)$ & $11(55 \%)$ & $4(20 \%)$ & $60(45 \%)$ \\
\hline$\geq 40$ years & $35(48 \%)$ & $13(65 \%)$ & $9(45 \%)$ & $16(80 \%)$ & $73(55 \%)$ \\
\hline \multicolumn{6}{|l|}{ Side } \\
\hline Left & $36(49 \%)$ & $9(45 \%)$ & $8(40 \%)$ & $6(30 \%)$ & $59(45 \%)$ \\
\hline Right & $37(51 \%)$ & $11(55 \%)$ & $12(60 \%)$ & $14(70 \%)$ & $74(55 \%)$ \\
\hline
\end{tabular}

to deep seated leasons. Two independent neuropathologists performed the histopathological diagnosis and grading based on the WHO classification 2007. The study was approved by the local ethics committee (Application No. 03-170).

RNA extraction and quantitative real-time PCR. RNA from frozen human tumor samples was isolated with the RNeasy Kit (Qiagen, Hilden, Germany) and the cDNA-synthesization performed with the QuantiTect Reverse Transcription Kit (Qiagen, Hilden, Germany). Quantitative real-time polymerase chain reaction (PCR) was performed with Cycler Rotor-Gene $Q$ and the Rotor-Gene SYBR Green PCR Kit (Qiagen, Hilden, Germany). Each run was carried out in triplicate with a reaction volume of $15 \mu \mathrm{l}$ with a two-step protocol $\left(95^{\circ}\right.$ for $5 \mathrm{~min} / 95^{\circ}$ for 5 seconds $/ 60^{\circ}$ for $\left.10 \mathrm{sec}\right)$ and 45 repetitions. Succinate dehydrogenase complex, subunit A, flavoprotein variant (SDHA) was used as housekeeping gene. The expression level was calculated by dividing the value of FAS or FAS-L by the value of SDHA. The primers used were: SDHA (Qiagen, Hilden, Germany, QT0059486), FAS (Qiagen, Hilden, Germany, QT00030618) and FAS-L (Eurofins, Luxemburg, SenseTGGAATTGTCCTGCTTTCTGG, Antisense-TGTTGCAAGATTGACCCCG).

Immunohistology. Tumors were frozen, cut, mounted on slides and fixed with paraformaldehyde and acetone. Tris-buffered saline with Tween (TBST) was used for washing. The primary antibodies were obtained from antibodies-online.de (FASL, ABIN223056) and Abcam, Cambridge, UK, (FAS, ab2437) and incubated in phosphate buffered saline (PBS) with $1 \%$ bovine serum albumin (BSA) and $3 \%$ Triton overnight at $4{ }^{\circ} \mathrm{C}$. The secondary antibodies (anti-mouse and anti-rabbit, biotinylated) were incubated in PBS with $1 \%$ BSA for 2 hours. Hemalaun was used to stain the nuclei. The VECTASTAIN ${ }^{\circledR}$ Elite ABC HRP Kit (Peroxidase, Standard) was used to bind the secondary antibodies. The ImmPACT DAB substrate Kit (SK-4105) was used for staining with Diaminobenzidine (DAB).
IDH1 mutation analysis. IDH1 mutations were detected by PCR and direct sequencing of amplified cDNA. The IDH1 primers (SenseGTGCCACTATCACTCCTGATG, Antisense-AAGGCCAACCC TTAGACAGAG) were designed with Primer3 v.0.4.0 (http:// bioinfo.ut.ee/primer3-0.4.0/). The PCR products were purified by Exo/SAP digestion with Exonuclease I (New England Biolabs, Beverly, MA) and Shrimp Alkaline Phosphatase (Promega, San Diego, USA). Sequencing was done with the ABI PRISM BigDye ${ }^{\circledR}$ Terminator v1.1 Cycle Sequencing Kit and the ABI 3730 sequencing instrument (Applied Biosystems, Foster City, CA, USA) following the manufacturer's instructions.

Statistical analyses. Data analysis was performed with GraphPad Prism 7. In the group comparison, Grubbs' test (alpha 0.05) was used to identify outliers. Kruskal-Wallis test was performed with one-way Analysis of variance (ANOVA) and Dunn's multiple comparisons test to identify statistically significant differences. High and low expression was compared in Kaplan-Meier plots by subdividing the patients into two groups according to higher or lower expression compared to the median of the group. Comparison of survival curves was performed with the Log-rank (Mantel-Cox) test.

\section{Results}

Expression level of FAS and FAS-L in gliomas. Table I summarises the baseline demographic and disease-specific characteristics. To illustrate FAS and FAS-L expression in gliomas, we collected 133 samples consisting of 73 grade II gliomas and each 20 samples from grade III gliomas, secondary and primary GBM. The expression level of FAS was highest in primary GBM (mean: 11.36 , 95\% CI=7.7714.94) and lowest in low-grade gliomas (mean: 1.75, 95\% CI=1.45-2.06) (Figure 1). Low-grade gliomas had 


\section{FAS and FASL expression in glioma and control tissue}
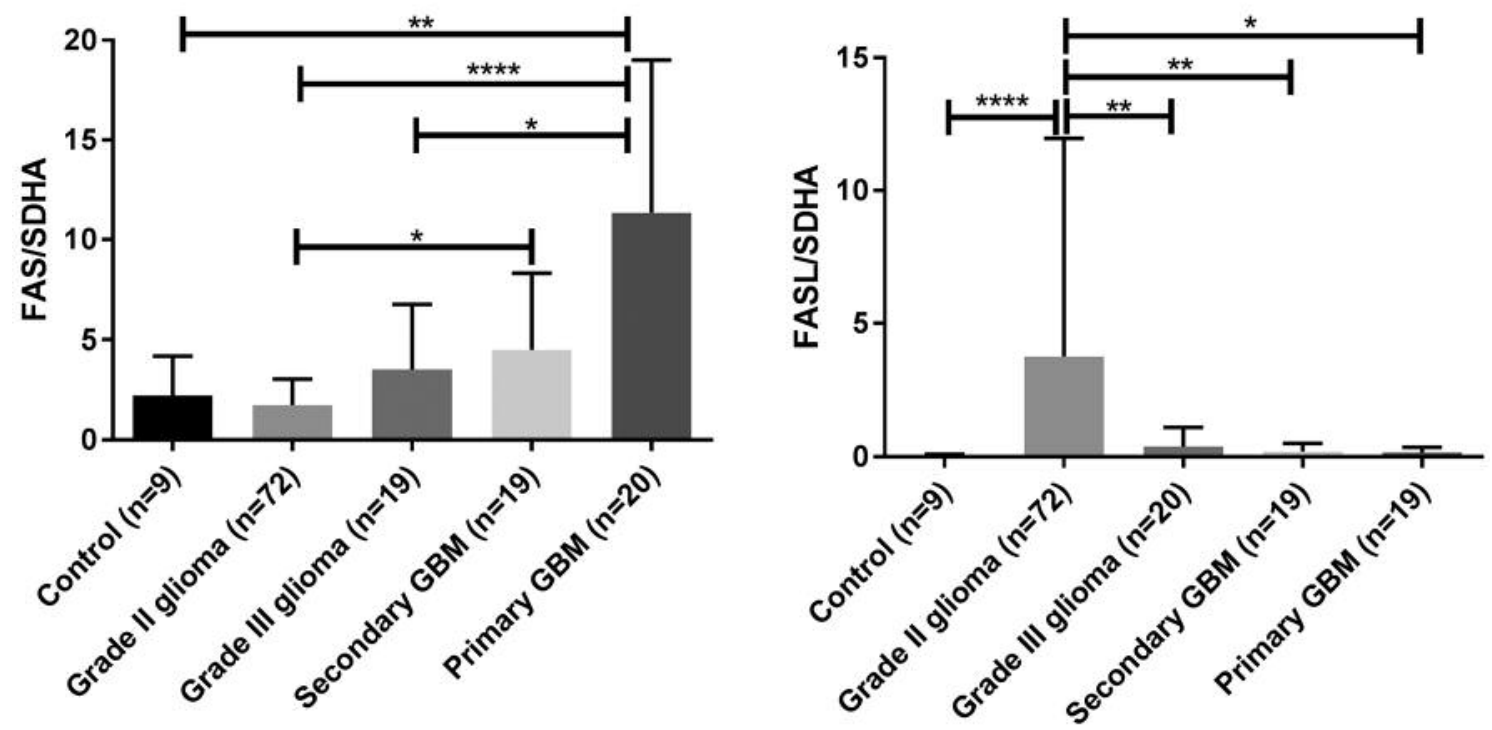

Figure 1. Comparison of FAS (left) and FAS-L (right) expression levels in gliomas (WHO grade II to IV) and control non-tumorous tissue. Statistical significance is marked with $* p<0.05, * * p<0.01$ and $* * * p<0.001$. Columns display the mean values and error bars the standard deviation.

statistically significantly lower levels of FAS compared to secondary- and primary GBM with $p<0.01$ and $p<0.001$ respectively. FAS-L levels were higher in low-grade gliomas (mean: $3.78,95 \% \mathrm{CI}=1.86-5.71$ ) compared to non-tumorous control tissue (mean: $0.04,95 \% \mathrm{CI}=0.00-0.08$ ), grade III gliomas (mean: $0.38,95 \% \mathrm{CI}=0.04-0.72$ ) and both, primary(mean: $0.16,95 \% \mathrm{CI}=0.07-0.25$ ) and secondary GBM (mean: $0.19,95 \% \mathrm{CI}=0.04-0.34)$.

Immunohistochemistry. Immunostaining showed increasing expression of FAS from low-grade gliomas to secondary glioblastomas (Figure 2). Secondary GBM showed strong staining of FAS in perivascular areas. Primary GBM presented the most intense staining of FAS. Only little FASL expression was detected in all tissue, except low-grade tumors, which showed several areas of intense FASL staining (Figure 2).

FAS and FAS-L in low-grade gliomas. In total, 73 samples of grade II (GII) gliomas were analyzed (Table II). Fifteen of these cases were based on the same patient with samples of the primary and the recurrent tumor. The total number of patients was 58. Non-tumorous tissue was collected from 10 other patients. The comparison of the expression level of FAS and FAS-L in primary- and relapse GII gliomas is shown in Figure 3a. FAS-L expression was significantly higher in the primary- (mean: $4.87,95 \% \mathrm{CI}=2.14-7.59$ ) and relapse (mean: 1.03, 95\% CI=0.05-2.01) GII gliomas compared to peritumoral control tissue (mean: $0.04,95 \% \mathrm{CI}=0.00-0.08$ ) with $p<0.001$ and $p<0.05$, respectively. FAS-L expression was significantly lower in relapsed- compared to primary GII gliomas $(p<0.05)$. No statistically significant differences in FAS or FAS-L expression were found between classical histologies of low-grade astrocytoma, oligoastrocytoma and oligodendroglioma (Figure 3b).

Matched cases of primary-and relapsed low-grade gliomas. The individual cases showed a rise of FAS expression in 9 and a fall of FAS-L expression in 7 out of the 15 matched cases. In this context, there was no trend toward concordant development of both FAS and FAS-L to simultaneously rise or fall. The detailed list of individual development within the longitudinal course of the disease is presented in Table III.

FAS and FAS-L as prognostic marker. We identified 61 patients with an IDHI mutation. The IDHI mutation did not show a significant influence on the expression of either FAS or FAS-L as illustrated in Figure 4a. Also, all other known relevant prognostic markers were analysed regarding lowand high FAS-L expression (Table IV). No significant difference was found between the groups, indicating equally balanced groups for the survival analysis between high and low FAS/FAS-L (see below).

The Prognostic relevance of FAS/FAS-L was tested twofold: i. type of progression, ii. survival. The group of low-grade gliomas was divided into a malignant and a stable 


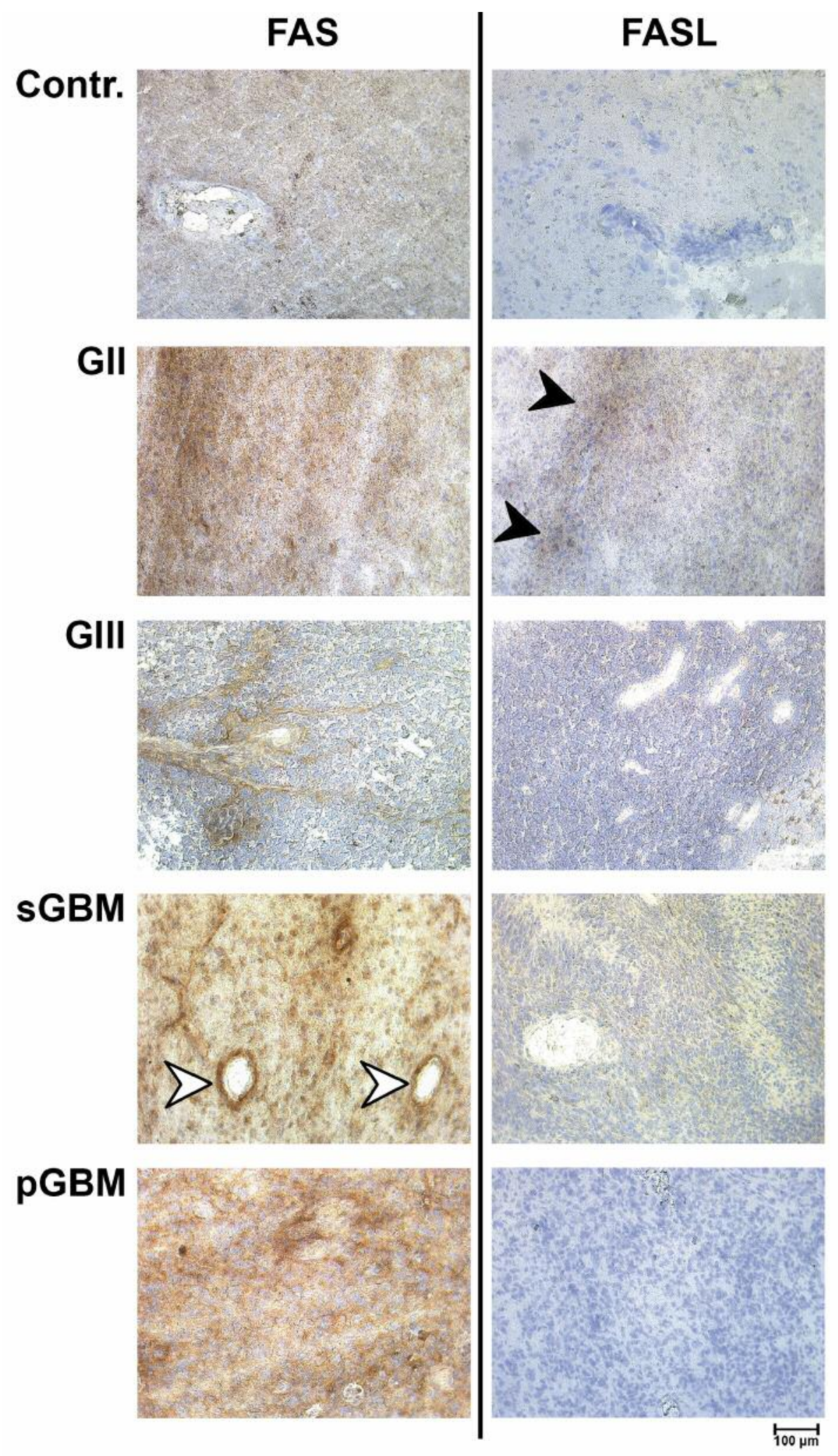

Figure 2. Immunostaining of non-tumorous tissue (Contr.), grade II glioma (GII), grade III glioma (GIII), secondary glioblastoma (sGBM) and primary glioblastoma ( $p G B M)$ visualized by Diaminobenzidine (DAB) and antibodies against FAS (left) and FASL (right). The cell nuclei were stained with hemalaun (blue). Empty arrows indicate perivascular staining (FAS) and filled arrows area of FASL expression in low-grade gliomas. Immunostaining of FAS and FASL reflects our data from real-time PCR with the highest expression/staining of FAS in primary GBM and FASL in low-grade gliomas. 
Table II. Detailed clinical data on grade II glioma patients: Summary of patient-specific data of 73 low-grade glioma samples included in the study. The samples are divided into primary $(n=43)$ and secondary $(n=30)$ low-grade gliomas. The data features: histology, sex, age, tumour location, side, Karnofsky performance score (KPS), IDH1 mutation status, chemo-/radiotherapy and mean survival.

\begin{tabular}{|c|c|c|c|c|c|}
\hline & \multicolumn{2}{|c|}{ Primary GII glioma } & \multicolumn{2}{|c|}{ GII glioma relapse } & \multirow{2}{*}{$\begin{array}{c}\text { Total } \\
\text { Samples (\%) }\end{array}$} \\
\hline & Samples & $\%$ & Samples & $\%$ & \\
\hline Number & 43 & $59 \%$ & 30 & $41 \%$ & $73(100 \%)$ \\
\hline \multicolumn{6}{|l|}{ Histology } \\
\hline Astrocytoma & 21 & $49 \%$ & 13 & $43 \%$ & $34(47 \%)$ \\
\hline Oligoastrocytoma & 16 & $37 \%$ & 12 & $40 \%$ & $28(38 \%)$ \\
\hline Oligodendroglioma & 6 & $14 \%$ & 5 & $17 \%$ & $11(15 \%)$ \\
\hline \multicolumn{6}{|l|}{ Gender } \\
\hline Female & 20 & $47 \%$ & 14 & $47 \%$ & $34(47 \%)$ \\
\hline Male & 23 & $53 \%$ & 16 & $53 \%$ & $39(53 \%)$ \\
\hline \multicolumn{6}{|l|}{ Age } \\
\hline$<40$ years & 28 & $65 \%$ & 10 & $33 \%$ & $38(52 \%)$ \\
\hline$\geq 40$ years & 15 & $35 \%$ & 20 & $67 \%$ & $35(48 \%)$ \\
\hline \multicolumn{6}{|l|}{ Tumour location } \\
\hline Frontal & 21 & $49 \%$ & 12 & $40 \%$ & $33(45 \%)$ \\
\hline Temporal & 7 & $16 \%$ & 11 & $37 \%$ & $18(25 \%)$ \\
\hline Parietal & 1 & $2 \%$ & 1 & $3 \%$ & $2(3 \%)$ \\
\hline Occipital & 2 & $5 \%$ & 0 & $0 \%$ & $2(3 \%)$ \\
\hline Parieto-occipital & 1 & $2 \%$ & 2 & $7 \%$ & $3(4 \%)$ \\
\hline Other & 11 & $26 \%$ & 4 & $13 \%$ & $15(21 \%)$ \\
\hline \multicolumn{6}{|l|}{ Side } \\
\hline Left & 20 & $47 \%$ & 16 & $53 \%$ & $36(49 \%)$ \\
\hline Right & 23 & $53 \%$ & 14 & $47 \%$ & $37(51 \%)$ \\
\hline \multicolumn{6}{|l|}{ KPS (before Surgery) } \\
\hline $100-80$ & 31 & $72 \%$ & 18 & $60 \%$ & $49(67 \%)$ \\
\hline$<80$ & 2 & $5 \%$ & 4 & $13 \%$ & $6(8 \%)$ \\
\hline No data & 10 & $23 \%$ & 8 & $27 \%$ & $18(25 \%)$ \\
\hline Mean KPS & 90 & 83 & 87 & & \\
\hline \multicolumn{6}{|l|}{$I D H 1$} \\
\hline IDH1 mutation & 38 & $88 \%$ & 23 & $82 \%$ & $61(86 \%)$ \\
\hline Wildtype & 5 & $12 \%$ & 5 & $18 \%$ & $10(14 \%)$ \\
\hline \multicolumn{6}{|l|}{ Radiotherapy } \\
\hline Radiotherapy & 7 & $16 \%$ & 3 & $9 \%$ & $10(14 \%)$ \\
\hline Stereotactic Brachytherapy & 7 & $16 \%$ & 8 & $24 \%$ & $15(21 \%)$ \\
\hline Radio-Brachytherapy & 1 & $2 \%$ & 1 & $3 \%$ & $2(3 \%)$ \\
\hline No Radiotherapy & 19 & $44 \%$ & 9 & $27 \%$ & $28(38 \%)$ \\
\hline No data & 9 & $21 \%$ & 9 & $27 \%$ & $18(25 \%)$ \\
\hline \multicolumn{6}{|c|}{ Mean survival in months $(95 \% \mathrm{CI})$} \\
\hline Progression free survival & \multicolumn{2}{|c|}{$51.19(37.73-64.66)$} & \multicolumn{2}{|c|}{$40.34(24.57-56.11)$} & $48(38-59)$ \\
\hline Overall survival & \multicolumn{2}{|c|}{$125.2(103.3-147.1)$} & \multicolumn{2}{|c|}{$97.23(72.6-121.9)$} & $113(97-130)$ \\
\hline
\end{tabular}

progression group: the malignant group $(n=33)$ with patients who relapsed with a higher WHO grade such as grade III or IV and the stable group $(n=25)$ with patients who relapsed again as grade II gliomas. No statistically significant difference in the expression of either FAS or FAS-L was found between the two groups (Figure 4b).

The potential prognostic relevance of FAS or FAS-L expression was assessed (Figures 5 and 6). Patients with high expression of FAS-L (mRNA expression above the median expression level) showed longer progression-free survival with a median survival of $46.50 v s .29 .93$ months $(p=0.0016$,
Figure 5b). As the expression of FAS-L differs from primary to relapsed low-grade gliomas, survival analysis was performed again individually (Figure 6). High FAS-L expression had a statistically significant advantage for the progression-free survival in primary low-grade gliomas with a median survival of $45.20 v s .31 .37$ months $(p<0.0062$, Figure 6a). No difference for FAS-L was seen in relapsed tumors. The expression of FAS did not show any significant difference in overall or progression-free survival (Figure 5a). Regarding overall survival, no statistically significant influence was found. 
A

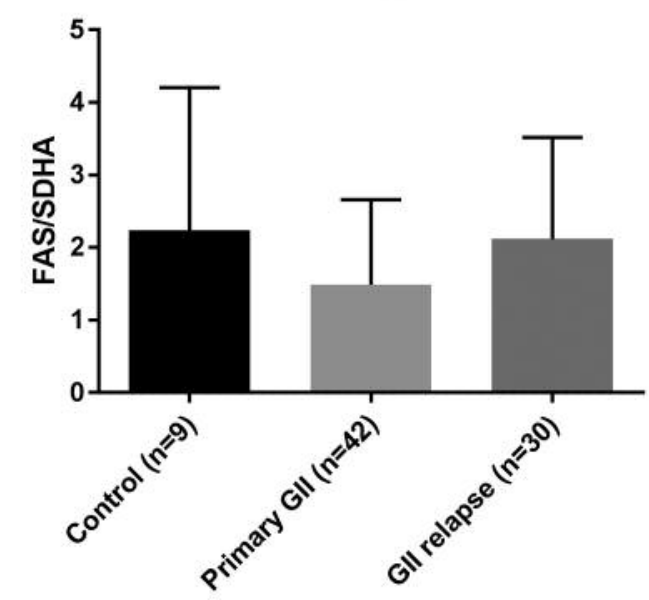

B

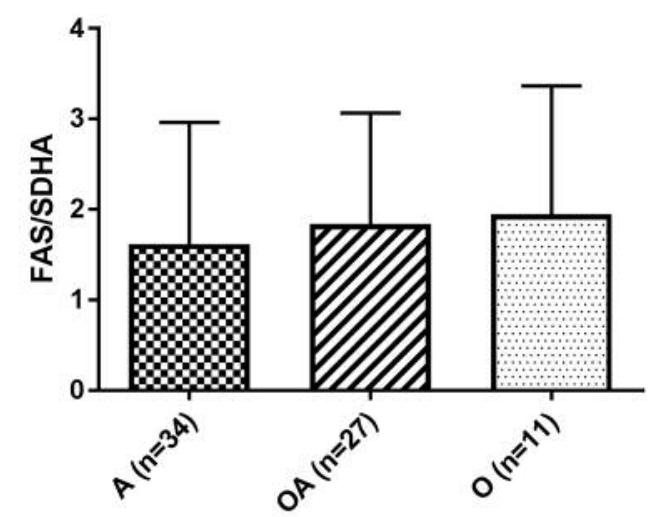

FASL

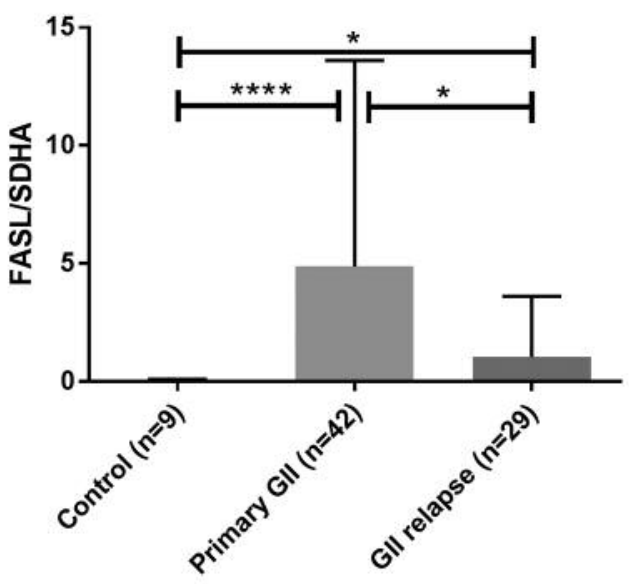

FASL

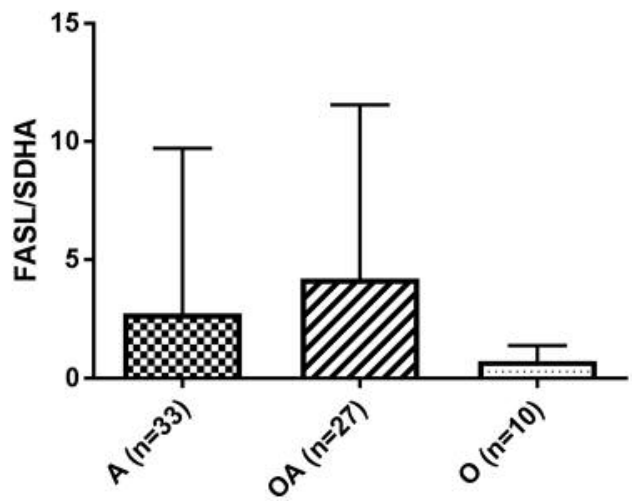

Figure 3. Comparison of FAS and FAS-L expression levels in primary- and relapse GII gliomas and control non-tumorous tissue (A) and astrocytoma, oligoastrocytoma and oligodendroglioma $(B)$. Statistical significance is marked with * $(p<0.05)$ and $* * * *(p<0.001)$. Columns display the mean values and error bars the standard deviation. A: Astrocytoma; OA: oligoastrocytoma; O: oligodendroglioma; GII: WHO grade II glioma.

\section{Discussion}

Our data show a statistically significant higher expression of FAS-L in low-grade gliomas compared to control tissue and high-grade gliomas. The study of Ehrmann, Rihakova (14) supports our findings in a study on apoptosis-related proteins (including FAS and FAS-L) in gliomas. They indicated higher FAS-L expression in low-grade astroglial tumors compared to high-grades in a semiquantitative manner. This is the first study showing a FAS-L dependent statistically significant benefit in the progression-free survival in primary low-grade gliomas (45.20 vs. 31.37 months).

In contrast, we observed no statistically significant correlation of FAS expression and overall- or progression-free survival data in low-grade gliomas. Our findings are in line with the data of Ehrmann, Rihakova (14) and the report of Frankel, Longo (15) on FAS expression in high-grade gliomas. The latter study showed a significant correlation of FAS expression with the apoptotic index, but no significantly longer median survival of grade III gliomas and GBM with higher FAS expression (20 vs. 18 months and 9 vs. 7.4 months).

Our study is, to our knowledge, the first evaluation of FAS and FAS-L expression in the comparison of primary and relapsed low-grade gliomas. We showed significantly higher levels of FAS-L in primary low-grade gliomas. A statistically not significant trend towards higher FAS expression in relapsed low-grade gliomas was visible. The findings of whole group comparison are supported by the comparison of the individual courses of patients with samples of both primary- and relapsed gliomas. 
A

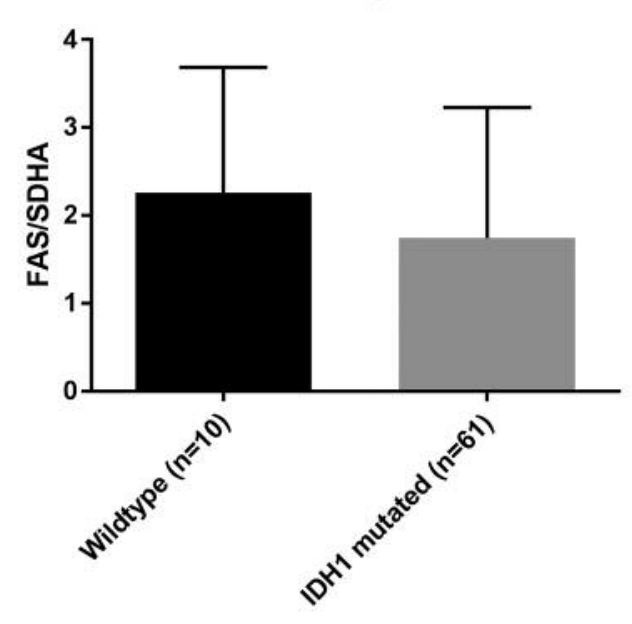

B

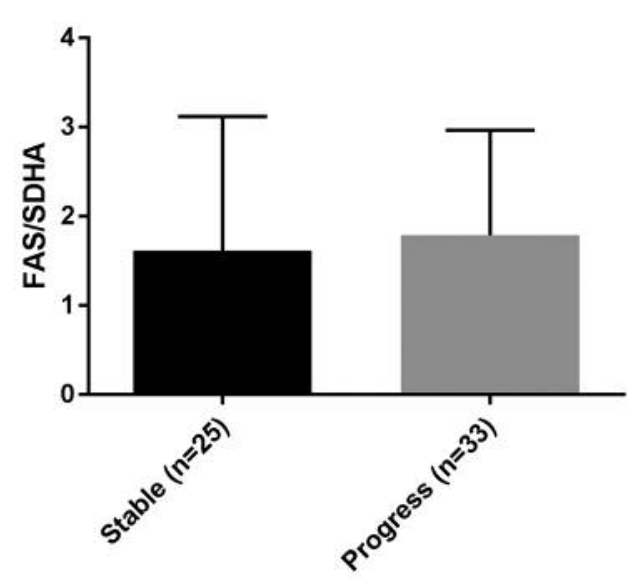

FASL

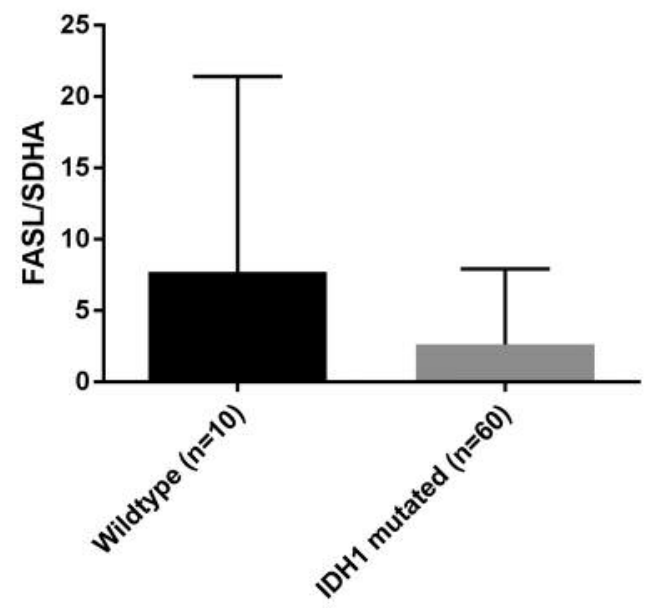

FASL

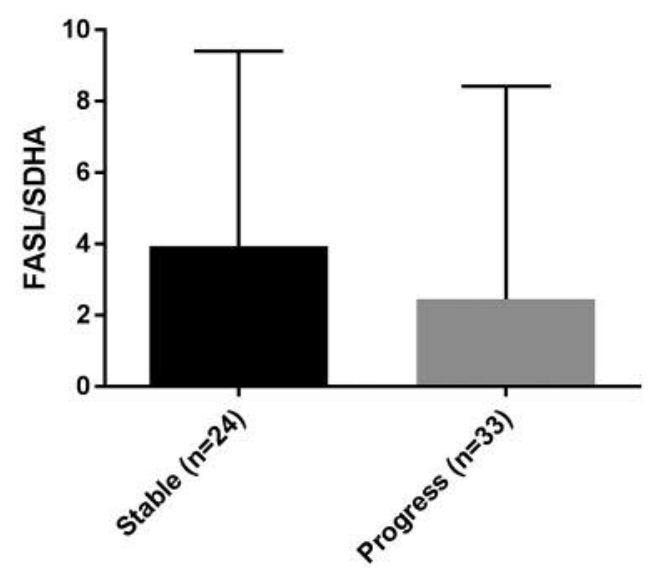

Figure 4. Comparison of FAS and FAS-L expression levels in wildtype-and IDH1 mutated low-grade gliomas (A); stable and progressive low-grade gliomas (B). Columns display the mean values and error bars the standard deviation.

The FAS/FAS-L pathway in clinical trials. The phase II study (NCT01071837) on recurrent GBM with the application of the FAS-L binding fusion protein APG101 and reirradiation by Wick, Fricke (16) indicates targeting the FAS/FAS-L pathway as a concept with a benefit in the progression-free survival. APG101 is well tolerated, as attested by the phase Ia study of Tuettenberg, Seiz (17). Currently a Phase I study (NCT02853565) is recruiting for the first trial on CAN008, an FAS-L targeting agent, in newly diagnosed GBM.

Implications for further studies. First of all, the prognostic importance of FAS-L regarding progression-free survival in primary low-grade gliomas should be confirmed in larger series. Our findings of high levels of FAS-L in low-grade glioma and differing levels of FAS-L expression in primary and secondary low-grade gliomas should be taken into consideration in further studies. Eventually, clinical trial activities targeting the FAS/FAS-L pathway should be extended from GBM to low-grade gliomas because FAS(-L) seems to be an important antigen in LGG and could therefore be a potential target irrespective of the prognostic value.

\section{Conclusion}

Our results show three major findings: Low-grade gliomas have lower FAS- and higher FAS-L expression compared to high-grade gliomas. The FAS-L expression is higher in primary compared to relapsed low-grade gliomas. FAS-L is a beneficial prognostic marker in primary low-grade gliomas regarding progression-free survival. 
A

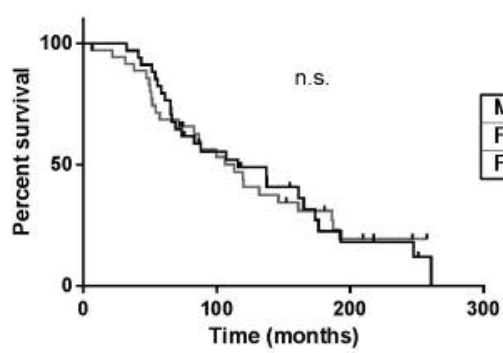

B

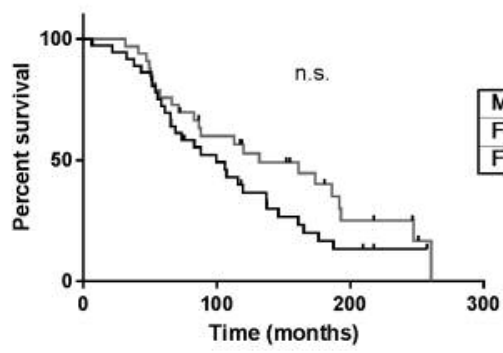

FAS

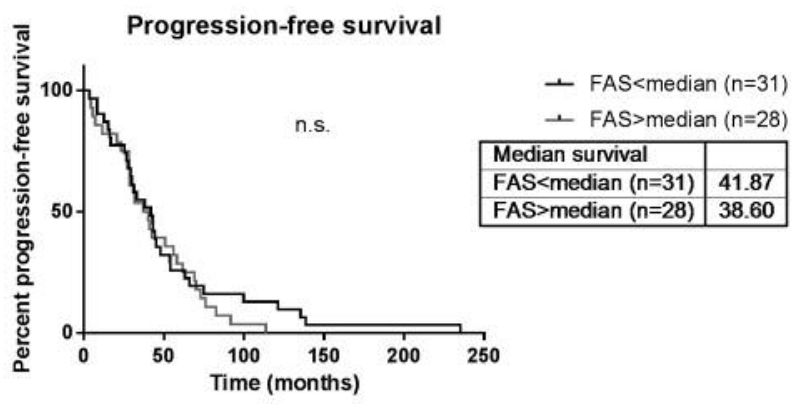

FAS-L

- FASL <median $(n=37)$

- FASL $>$ median $(n=33)$

\begin{tabular}{l|l}
\hline Median survival & \\
FASL $<$ median $(n=37)$ & 99.50 \\
\hline
\end{tabular}

FASL $>$ median $(n=33) \quad 132.0$

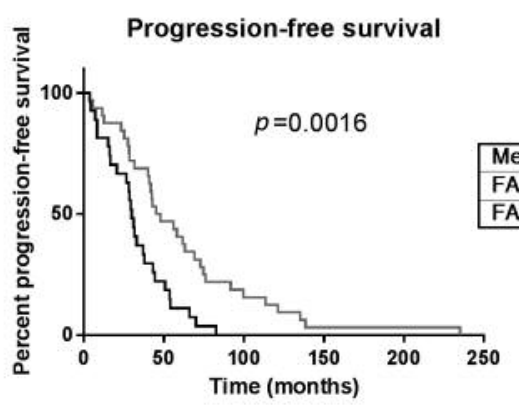

- FASL $<$ median $(n=27)$

- FASL $>$ median $(n=32$

\begin{tabular}{|l|l|} 
Median survival & \\
FASL $<$ median $(n=27)$ & 29.93 \\
\hline
\end{tabular}

FASL $>$ median $(n=32) \quad 46.50$

Figure 5. Kaplan-Meier plots of the overall (left) and progression-free (right) survival according to FAS expression (A) and FASL expression (B).

FAS-L

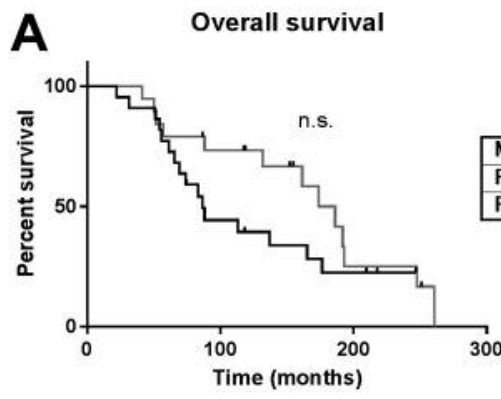

Primary low-grade gliomas

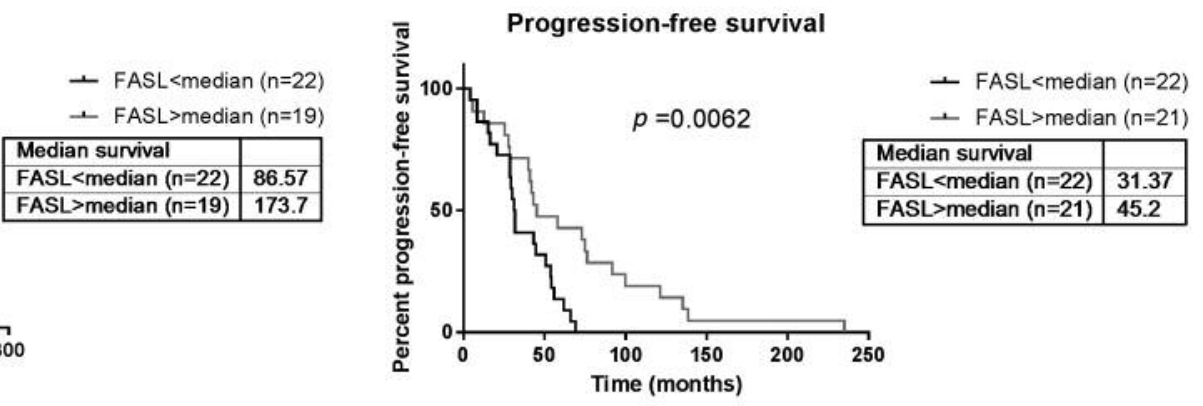

\section{Relapsed low-grad gliomas}

B

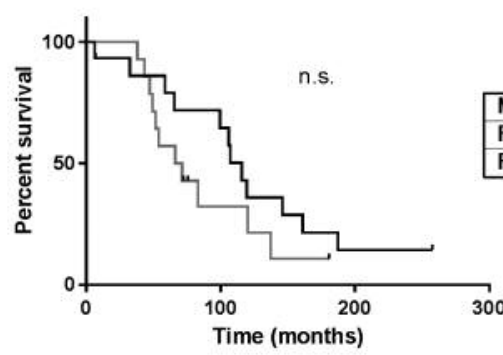

Progression-free survival

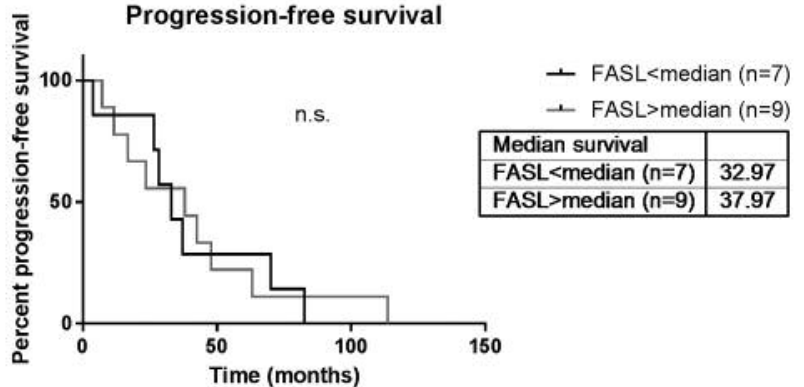

Figure 6. Kaplan-Meier plots of overall (left) and progression-free (right) survival according to FAS-L expression in primary (A) and relapsed (B) low-grade gliomas. 
Table III. Individual cases: Longitudinal course of the disease within the same patient: individual development of FAS and FAS-L expression in matched cases of primary-and relapsed low-grade glioma patients ( $\boldsymbol{\uparrow}$ severe rise, $\boldsymbol{\Downarrow}$ severe fall, $\boldsymbol{\lambda}$ rise, $\boldsymbol{\mathbf { y }}$ fall, and $\rightarrow$ constant expression).

\begin{tabular}{llccc}
\hline Patient ID & & $\begin{array}{c}\text { Primary low-grade } \\
\text { glioma }\end{array}$ & $\begin{array}{c}\text { Low-grade } \\
\text { glioma relapse }\end{array}$ & Tendency \\
\hline 2894 & FAS & 0.63 & 1.53 & $\boldsymbol{\lambda}$ \\
& FAS-L & 3.51 & 1.78 & $\mathbf{y}$ \\
1461 & FAS & 4.94 & 4.15 & $\mathbf{y}$ \\
& FAS-L & 0.58 & 13.31 & $\boldsymbol{\uparrow}$ \\
697 & FAS & 3.95 & 2.45 & $\mathbf{y}$ \\
& FAS-L & 15.46 & 0.02 & $\boldsymbol{\Downarrow}$ \\
2141 & FAS & 3.87 & 4.19 & $\boldsymbol{\lambda}$ \\
& FAS-L & 0.97 & 0.95 & $\mathbf{\rightarrow}$ \\
539 & FAS & 0.15 & 1.29 & $\boldsymbol{\lambda}$ \\
& FAS-L & 3.36 & 38.13 & $\boldsymbol{\uparrow}$ \\
663 & FAS & 0.36 & 0.39 & $\mathbf{\rightarrow}$ \\
375 & FAS-L & 0.02 & 0.02 & $\mathbf{7}$ \\
& FAS & 0.34 & 2.54 & $\mathbf{\lambda}$ \\
3219 & FAS-L & 0.24 & 0.04 & $\mathbf{y}$ \\
& FAS & 1.00 & 0.62 & $\mathbf{y}$ \\
& FAS-L & 2.44 & 0.51 & $\mathbf{y}$ \\
\hline
\end{tabular}

\begin{tabular}{|c|c|c|c|c|}
\hline Patient ID & & $\begin{array}{c}\text { Primary low-grade } \\
\text { glioma }\end{array}$ & $\begin{array}{l}\text { Low-grade } \\
\text { glioma relapse }\end{array}$ & Tendency \\
\hline \multirow[t]{2}{*}{665} & FAS & 1.24 & 2.97 & $\pi$ \\
\hline & FAS-L & 0.12 & 0.12 & $\rightarrow$ \\
\hline \multirow[t]{2}{*}{469} & FAS & 1.82 & 1.15 & $y$ \\
\hline & FAS-L & 7.24 & 0.20 & $\downarrow$ \\
\hline \multirow[t]{2}{*}{2915} & FAS & 0.23 & 0.44 & $\pi$ \\
\hline & FAS-L & 8.32 & 0.57 & $y$ \\
\hline \multirow[t]{2}{*}{335} & FAS & 0.21 & 2.26 & $\pi$ \\
\hline & FAS-L & 5.06 & 0.04 & $y$ \\
\hline \multirow[t]{2}{*}{1109} & FAS & 4.21 & 1.28 & $y$ \\
\hline & FAS-L & 0.04 & 0.03 & $\rightarrow$ \\
\hline \multirow[t]{2}{*}{369} & FAS & 0.65 & 3.07 & $\pi$ \\
\hline & FAS-L & 0.03 & 0.16 & $\rightarrow$ \\
\hline \multirow[t]{2}{*}{401} & FAS & 0.94 & 4.31 & $\pi$ \\
\hline & FAS-L & 0.09 & 0.81 & $\pi$ \\
\hline
\end{tabular}

Table IV. Group comparison of high- and low-FasL expression in primary GII glioma: Summary of the patient specific data of 43 primary lowgrade glioma samples divided into low- $(n=22)$ and high- $(n=21)$ FasL expression. Pearsons Chi-Square test shows no significant (n.s.) difference between both groups regarding: age, sex, KPS, Histology, IDH1-mutation, operation results or radiotherapy. Data on chemotherapy was available in 24 cases, of which none received chemotherapy.

\begin{tabular}{|c|c|c|c|c|c|c|c|}
\hline & \multicolumn{3}{|c|}{ Low FasL } & \multicolumn{3}{|c|}{ High FasL } & \multirow{2}{*}{$\begin{array}{c}\text { Chi-Square } \\
p \text {-Value }\end{array}$} \\
\hline & Samples & $\%$ (Row) & $\%$ (Column) & Samples & $\%$ (Row) & $\%$ (Column) & \\
\hline Number & 22 & & & 21 & & & \\
\hline \multicolumn{8}{|l|}{ Age } \\
\hline$<40$ years & 14 & $51.9 \%$ & $63.6 \%$ & 13 & $48.1 \%$ & $61.9 \%$ & \multirow[t]{3}{*}{ n.s. $(0.907)$} \\
\hline$>40$ years & 8 & $50.0 \%$ & $36.4 \%$ & 8 & $50.0 \%$ & $38.1 \%$ & \\
\hline mean & & 39.05 & & & 39.05 & & \\
\hline \multicolumn{8}{|l|}{ Gender } \\
\hline Female & 9 & $45.0 \%$ & $40.9 \%$ & 11 & $55.0 \%$ & $52.4 \%$ & \multirow[t]{2}{*}{ n.s. $(0.451)$} \\
\hline Male & 13 & $56.5 \%$ & $59.1 \%$ & 10 & $43.5 \%$ & $47.6 \%$ & \\
\hline Karnofsky Performance Score & & Mean: 92 & & & Mean: 87 & & n.s. $(0.500)$ \\
\hline No data & 2 & 7 & & & & & \\
\hline \multicolumn{8}{|l|}{ Histology } \\
\hline Astrocytoma & 13 & $61.9 \%$ & $59.1 \%$ & 8 & $38.1 \%$ & $38.1 \%$ & \multirow[t]{3}{*}{ n.s. $(0.335)$} \\
\hline Oligoastrocytoma & 6 & $37.5 \%$ & $27.3 \%$ & 10 & $62.5 \%$ & $47.6 \%$ & \\
\hline Oligodendroglioma & 3 & $50.0 \%$ & $13.6 \%$ & 3 & $50.0 \%$ & $14.3 \%$ & \\
\hline \multicolumn{8}{|l|}{ IDH1 } \\
\hline IDH1 wildtype & 4 & $57.1 \%$ & $18.2 \%$ & 3 & $42.9 \%$ & $14.3 \%$ & \multirow[t]{2}{*}{ n.s. (0.729) } \\
\hline IDH1 mutated & 18 & $50.0 \%$ & $81.8 \%$ & 18 & $50.0 \%$ & $85.7 \%$ & \\
\hline \multicolumn{8}{|l|}{ OP-Results } \\
\hline STR & 7 & $50.0 \%$ & $31.8 \%$ & 7 & $50.0 \%$ & $33.3 \%$ & \multirow[t]{3}{*}{ n.s. $(0.249)$} \\
\hline GTR & 11 & $64.7 \%$ & $50.0 \%$ & 6 & $35.3 \%$ & $28.6 \%$ & \\
\hline No data & 4 & $33.3 \%$ & $18.2 \%$ & 8 & $66.7 \%$ & $38.1 \%$ & \\
\hline \multicolumn{8}{|l|}{ Radiotherapy } \\
\hline None & 11 & $57.9 \%$ & $50.0 \%$ & 8 & $42.1 \%$ & $38.1 \%$ & \multirow[t]{3}{*}{ n.s. $(0.135)$} \\
\hline Radio-/Brachytherapy & 9 & $60.0 \%$ & $40.9 \%$ & 6 & $40.0 \%$ & $28.6 \%$ & \\
\hline No data & 2 & $22.2 \%$ & $9.1 \%$ & 7 & $77.8 \%$ & $33.3 \%$ & \\
\hline
\end{tabular}




\section{Acknowledgements}

This work was supported by a grant from the German Foundation for Young Adults with Cancer (to J. Werner).

\section{References}

1 Ho VK, Reijneveld JC, Enting RH Bienfait HP, Robe P, Baumert BG, Visser O and Dutch Society for Neuro-Oncology (LWNO): Changing incidence and improved survival of gliomas. EurJ Cancer 50: 2309-2318, 2014.

2 Wen PY and Kesari S: Malignant gliomas in adults. New Engl J Med 359: 492-507, 2008.

3 Polivka J Jr., Polivka J, Holubec L, Kubikova T, Priban V, Hes $\mathrm{O}$, Pivovarcikova $\mathrm{K}$ and Treskova I: Advances in experimental targeted therapy and immunotherapy for patients with glioblastoma multiforme. Anticancer Res 37: 21-33, 2017.

4 Yan H, Parsons DW, Jin G, McLendon R, Rasheed BA, Yuan W, Kos I, Batinic-Haberle I, Jones S, Riggins GJ, Friedman H, Friedman A, Reardon D, Herndon J, Kinzler KW, Velculescu VE, Vogelstein B and Bigner DD: IDH1 and IDH2 mutations in gliomas. New Engl J Med 360: 765-773, 2009.

5 Ohgaki $\mathrm{H}$ and Kleihues $\mathrm{P}$ : The definition of primary and secondary glioblastoma. Clin Can Res 19: 764-772, 2013.

6 Pignatti F, van den Bent M, Curran D, Debruyne C, Sylvester R, Therasse P, Afra D, Cornu P, Bolla M, Vecht C, Karim AB, European Organization for Research and Treatment of Cancer Brain Tumor Cooperative Group and European Organization for Research and Treatment of Cancer Radiotherapy Cooperative Group: Prognostic factors for survival in adult patients with cerebral low-grade glioma. J Clin Oncol 20: 2076-2084, 2002.

7 Smith JS, Chang EF, Lamborn KR, Chang SM, Prados MD, Cha $\mathrm{S}$, Tihan T, Vandenberg S, McDermott MW and Berger MS: Role of extent of resection in the long-term outcome of lowgrade hemispheric gliomas. J Clin Oncol 26: 1338-1345, 2008.

8 Nagata S and Golstein P: The Fas death factor. Science 267: 1449-56, 1995.

9 Waring P and Mullbacher A: Cell death induced by the Fas/Fas ligand pathway and its role in pathology. Immunol Cell Biol 77: 312-317, 1999.
10 Itoh N, Yonehara S, Ishii A, Yonehara M, Mizushima S, Sameshima M, Hase A, Seto Y and Nagata S: The polypeptide encoded by the cDNA for human cell surface antigen Fas can mediate apoptosis. Cell 66: 233-243, 1991.

11 Eisele G, Wolpert F, Decrey G and Weller M: APO010, a synthetic hexameric CD95 ligand, induces death of human glioblastoma stem-like cells. Anticancer Res 33: 3563-3571, 2013.

12 Eisele G, Roth P, Hasenbach K, Aulwurm S, Wolpert F, Tabatabai G, Wick W and Weller M: APO010, a synthetic hexameric CD95 ligand, induces human glioma cell death in vitro and in vivo. Neuro-oncology 13: 155-164, 2011.

13 Tachibana O, Nakazawa H, Lampe J, Watanabe K, Kleihues P and Ohgaki H: Expression of Fas/APO-1 during the progression of astrocytomas. Cancer Res 55: 5528-5530, 1995.

14 Ehrmann J Jr., Rihakova P, Hlobilkova A, Kala M and Kolar Z: The expression of apoptosis-related proteins and the apoptotic rate in glial tumors of the brain. Neoplasma 47: 151-155, 2000.

15 Frankel B, Longo SL, Leach C, Canute GW and Ryken TC: Apoptosis and survival in high-grade astrocytomas as related to tumor Fas (APO-1/CD95) expression. J Neuro Oncol 59: 27-34, 2002.

16 Wick W, Fricke H, Junge K, Kobyakov G, Martens T, Heese O, Wiestler B, Schliesser MG, von Deimling A, Pichler J, Vetlova E, Harting I, Debus J, Hartmann C, Kunz C, Platten M, Bendszus $\mathrm{M}$ and Combs SE: A phase II, randomized, study of weekly APG101+reirradiation versus reirradiation in progressive glioblastoma. Clin Cancer Res 20: 6304-6313, 2014.

17 Tuettenberg J, Seiz M, Debatin KM, Hollburg W, von Staden M, Thiemann $M$, Hareng $B$, Fricke $H$ and Kunz $C$ : Pharmacokinetics, pharmacodynamics, safety and tolerability of APG101, a CD95-Fc fusion protein, in healthy volunteers and two glioma patients. Int Immunopharmacol 13: 93-100, 2012.

Received September 13, 2017

Revised September 26, 2017

Accepted September 27, 2017 